\title{
Investigation on Dielectric Constant of Zinc Oxide
}

\author{
Khalid Omar \\ School of Physics \\ Universiti Sains Malaysia \\ 11800 Penang, Malaysia \\ E-mail: khalhadithi@yahoo.com \\ M. D. Johan Ooi \& M. M. Hassin \\ School of Physics \\ Universiti Sains Malaysia \\ 11800 Penang, Malaysia
}

\begin{abstract}
The studies of the behavior of zinc oxide and its dielectric constant at different temperature ranging from $700{ }^{\circ} \mathrm{C}(975 \mathrm{~K})$ up to $1000{ }^{\circ} \mathrm{C}(1275 \mathrm{~K})$ have been done, as well as the fabrication of pellet. The pellets were sintered at $700{ }^{\circ} \mathrm{C}, 800{ }^{\circ} \mathrm{C}$, $900{ }^{\circ} \mathrm{C}$ and $1000{ }^{\circ} \mathrm{C}$, in a Nabertherm Controller Furnace and the heating rate is $6{ }^{\circ} \mathrm{C} / \mathrm{min}$. The capacitance and dissipation factor $(\tan \delta)$ were taken and calculated the relative permittivity, $\varepsilon_{\mathrm{r}}$ and dielectric loss, $\varepsilon_{\mathrm{i}}$ which are indicated the behavior and dielectric constant of zinc oxide that depend on different temperatures of sintered pellet and frequency of the applied LCR meter.
\end{abstract}

Keywords: Semiconductor, Zinc oxide, Dielectric constant

\section{Introduction}

Zinc oxide was the material of choice for this observation because it by far is the most common material used in the commercial manufacture and research due to its low cost and wide availability. It is valuable both for direct application or preparation of other compounds. Zinc oxide has been produced commercially for considerably more than century, originally for use as a pigment in paints and also for rubber, glass, porcelain enamels and pharmaceuticals. More recently, Zinc oxide has found new applications in semiconductors, luminescence, and photoconductivity. Under certain conditions, atoms of zinc in stoichio metric excess can lodge in these lacunae and this hypothesis is often useful in explaining certain specific properties of the oxide such as its semiconductivity, photoconductivity, luminescence, catalytic properties and solid state chemistry. It possess thermochromic which change color from white to yellow when heated, and back again to white color when cooled down due to various types of crystal lattice defects. It remains white when exposed to hydrogen sulfide or ultraviolet light. The larger red atoms are zinc atoms while the smaller purple atoms are oxygen atoms. In wurtzite, the tetrahedral are stacked in hexagonal close packed array with the tetrahedral edges of alternate layers rotated through $180^{\circ}$ about the c-axis. $\mathrm{ZnO}$ is almost insoluble in water but soluble in acids or alkalis. Its solubility is " $0.005 \mathrm{~g} / \mathrm{l}^{\prime}$ at $25^{\circ} \mathrm{C}$ in water while in aqueous ammonia its solubility is " $0.28 \mathrm{~g} / \mathrm{l}$ " in $1 \%$ ammonia at $25^{\circ} \mathrm{C}$. Its solubility is increased by the presence of ammonium salts [M. Farnsworth and C. H. Kline, (1973)]. Zinc oxide may be reduced by heating in hydrogen or carbon monoxide but it does not react with dry carbon dioxide. It is gradually changed to zinc oxycarbonate in humid air. It also does not react with oxygen to form peroxide. The band gap of $\mathrm{ZnO}$ can be tuned via divalent substitution on the action to produce heterostructures. Cadmium, $(\mathrm{Cd})$ doping can decrease the band gap to as low as $\sim 3.0 \mathrm{eV}$, whereas Magnesium (Mg) doping can increase the band gap to as high as $\sim 4.0 \mathrm{eV}$ [http://en.wikipedia.org/wiki/Zinc_oxide]. Electron doping in nominally undoped $\mathrm{ZnO}$ has been attributed to Zinc interstitials, oxygen vacancies, or hydrogen. The intrinsic defect levels lead to n-type doping lay approximately $0.01-0.05 \mathrm{eV}$ below the conduction band. Electrical properties of $\mathrm{ZnO}$ depend on the concentration of intentional impurities, called dopants. The dopants determine whether the current is carried by electrons or holes. In semiconducting oxides, it is generally possible to achieve one or other of these types, but not both. The dopants are also called shallow level impurities because they introduce energy levels close to one of the allowed energy bands in the 
material and are easily ionized as a result. There may also be unintentional impurities introduced during the growth of $\mathrm{ZnO}$ that have a deleterious effect on the properties of the material. These are called deep level defects or impurities and may be either elemental impurities arising from contamination of the growth environment or structural defects in the $\mathrm{ZnO}$ crystal lattice. These structural defects can be vacancies in the crystal structure or interstitials, i.e. atoms sitting in the open regions around lattice sites. In both cases, they may introduce energy levels deep within the forbidden band gap of $\mathrm{ZnO}$ and act as traps for carriers in the material. These uncontrolled defects make it very difficult to obtain reproducible device performance and reliability. For $\mathrm{ZnO}$, n-type conductivity is relatively easy to realize via excess Zinc or with Aluminum (Al), Gallium (Ga), or Indium (In) doping, but p-type doping has only recently been achieved. This is a fairly common occurrence in wide band gap semiconductors, where difficulty in achieving bipolar "n-type and p-type" doping is not unusual. This doping difficulty in wide bad gap semiconductors can be explained by compensation by native point defects or dopant atoms that locate on interstitial sites. The defect compensates for the substitution impurity level through the formation of a deep level trap. In some cases, strong lattice relaxations can drive the dopant energy level deeper within the gap. In other systems, one may simply have a low solubility for the chosen dopant, which limits the accessible extrinsic carrier density. In $\mathrm{ZnO}$, most candidate p-type dopants introduce deep acceptor levels. The most promising dopants for p-type material are the group V elements [D. P. Norton, et al (2004)]. Conductivity of $\mathrm{ZnO}$ is decreases when heated in oxygen under pressure and when heated in vacuum, its conductivity increases. Conductivity in the lower temperature range is believed to be caused by the ionization of interstitial zinc atom pairs whose ionization energy is $2 \times 10^{-2} \mathrm{~V}$ or less. The change in conductivity of Zinc oxide can also be caused by the addition of varying amounts of monovalent oxides decreases the conductivity whereas addition of trivalent oxides such as Aluminum (Al) or Chromium $(\mathrm{Cr})$ increases the conductivity. Zinc oxide is transparent to visible light to a relatively high and uniform degree so that it exhibits a pure white of high brilliance. It is opaque to ultra - violet radiation below $3655 \AA$ [M. Farnsworth et al (1973)]. Meanwhile the optical properties of ZnO, studied by using photoluminescence, photoconductivity, and absorption reflect the intrinsic direct band gap, a strongly bound exciton state, and gap states arising from point defects. A strong room temperature, near-band-edge ultraviolet (UV) photoluminescence peak at $\sim 3.2 \mathrm{eV}$ is attributed to an exciton state, as the exciton binding energy is on the order of 60 $\mathrm{meV}$. The high exciton binding energy is more resistant to radiation, and is multifunctional with uses in the areas as a piezoelectric [http://www.physorg.com/Zinc Oxide Nanostructures: Growth, Properties and Applications]. Zinc oxide is considered a better material than Gallium nitride $(\mathrm{GaN})$ for making blue LED devices because, it naturally emits and absorbs at $368 \mathrm{~nm}$ more efficiently and it is also cheap and abundant. The new LEDs will deliver 10 times the light-emitting efficiency. These LEDs are used as light sources in applications requiring high luminance, long service life and high efficiency, such as large outdoor displays, traffic signals, liquid-crystal display backlighting and illuminations [http://www.spectrum.ieee.org]. Zinc oxide continues as an essential ingredient in the "soft" type of ferromagnetic materials for television, radio, and telecommunication applications. In these fields ferrites based on Magnetite, Nickel oxide and Zinc oxide are used as elements in many types of electronic devices [http://www.navbharat.co.in]. The latest research about $\mathrm{ZnO}$ is that a team from Northwestern University had found that when they pumped disordered Zinc oxide powders with a conventional laser at low power, the materials gave off light with a broad band of wavelengths which the light in fact is a laser emission. The lasing wavelength is $380 \mathrm{~nm}$ and the devices operate at room temperature. If the lasing powder can be mass produced, they would be cheaper and more efficient than the LEDs now used as laser sources in cell phones, calculators and other devices with luminescent displays.

\section{Correlation between diffusion coefficient, mobility and conductivity}

Zinc oxide possesses a semiconductor behavior which it can be a dielectric at low temperature and being a conductor at high temperature. It is due to decreasing of energy band gap as temperature of the material increase but for this experiment, the factor that determines the behavior of $\mathrm{ZnO}$ is related to diffusion of atom during sintering process.

Zinc oxide contains a large number of grain boundaries, which represent a high- energy area because of the inefficient packing of the atoms. A lower overall energy is obtained in the material if the amount of grain boundary area is reduced by grain growth. Grain growth involves the movement of grain boundaries, permitting larger grains to grow at the expense of smaller grains. For grain growth in materials, diffusion of atoms across the grain boundary is required. In order to move to a new location, the atom must overcome an energy barrier which is the activation energy $Q$, and for that a thermal energy is required to exceed this barrier. The kinetics of process of diffusion is strongly dependent on temperature [D. R. Askeland et al (2005) ]. The diffusion coefficient $D$ is related to temperature by an Arrhenius- type equation,

$$
D=D_{0} \exp \left(\frac{-Q}{R T}\right)
$$

where $Q$ is the activation energy (cal/mol), T is the absolute temperature $(\mathrm{K}), D_{0}$ is Einstein diffusion constant and $R$ is gas constant which is $8.314(\mathrm{~J} / \mathrm{K} . \mathrm{mol})$. 
According "Equation 1", when the temperature of material increases, the diffusion coefficient $D$ increases and thermal energy supplied to the diffusing atoms permits the atoms to overcome the activation energy barrier and more easily move to another grain. High temperature increase diffusion coefficient. Existence of impurities also increases the electrical conductivity because it can diffuse and help carry the current. Therefore, at low temperature, the diffusion is slow, thus decreasing mobility and conductivity of the material [D. R. Askeland et al (2005)].

\subsection{Dielectric in term polarization and electric field}

$\mathrm{ZnO}$ crystal could possess a dielectric behavior when its energy barrier is large and its diffusion coefficient is low. Besides, the utmost important of $\mathrm{ZnO}$ is that it has built in polarization. Thus, it is important to know the basic of dielectric in term of polarization and electric field. In dielectric, all charges are attached to specific atoms or molecules and all they can do is move a bit within it. Their microscopic displacement, affects the characteristics behavior of dielectric materials. In any substance, including $\mathrm{ZnO}$ crystal, the atom is electrically neutral because there is a positively charged core "nucleus" and a negatively charged electron cloud surrounding it. These two regions of charge within the atom are influenced by the electric field. The nucleus is pushed in the direction of the field, and the electrons are pushed in the opposite ways. In principle, if the electric field is large enough, it can pull the atom apart completely, "ionizing" it. Thus, the substance then becomes a conductor. With less extreme electric fields, however, equilibrium is soon established. If the center of the electron cloud does not coincide with the nucleus, these positive and negative charges attract one another, and this holds the atoms together. The two opposing forces, electric field, $E$ pulling the electrons and nucleus apart, their mutual attraction drawing them together reach a balance, leaving the atom polarized, with plus charge shifted slightly one way and minus the other. In other words, dielectric polarization arises due to the existence of atomic and molecular forces, appears whenever charges in a material are displaced with respect to one another under the influence of an electric field [David J. Griffiths].

\section{Fabrication of Zinc oxide pellets}

The process involve in making $\mathrm{ZnO}$ pellets are the same with fabricating a ceramic. The involved procedures are Pulverization, Pressing and Sintering. The pulverization process is to reduce the particle size with the action of mechanical force. This is to provide a homogeneously mixture and has a sufficiently small particle size. The pressing is important in order to affect the sample in increasing the mechanical strength in order to make it more compact and Reducing void between particles. There are two techniques in this pressing process which are Isostatic Press and Hydraulic Press. Sintering is the critical process in fabricating ZnO pellets. It is commonly refers to processes involved in the heat treatment of powder compacts below its melting point until its particles adhere to each other where porosity is usually reduced and the grain size increases significantly through mass transport [R.M. German (1991)]. The sintering process is illustrated in the "Figure 1".

\section{Calculation of dielectric constant}

The dielectric constant, $\varepsilon$ is consists of real permittivity or relative dielectric constant which is generally called dielectric constant of the test material, $\varepsilon_{\mathrm{r}}$ and imaginary permittivity or dielectric loss $\varepsilon_{\mathrm{i}}$. Dielectric constant of the test material, $\varepsilon_{\mathrm{r}}$ is calculated from the measurement of capacitance value which can be obtained using the following equation,

$$
\varepsilon_{r}=\frac{t \times C_{p}}{A \times \varepsilon_{o}}=\frac{t \times C_{p}}{\pi\left(\frac{d}{2}\right)^{2} \times \varepsilon_{o}}
$$

where,

$$
t=\quad \text { Thickness of the pellet }
$$

$C_{p}=$ Equivalent parallel capacitance which obtained from the data of measurement

$$
\begin{gathered}
\varepsilon_{o}=\quad \text { permittivity of vacuum }=8.854 \times 10^{-12}(\mathrm{~F} / \mathrm{m}) \\
d=\quad \text { Diameter of guard electrode }=5 \times 10^{-3}(\mathrm{~m})
\end{gathered}
$$

Thus, the above equation becomes,

$$
\varepsilon_{r}=\frac{t \times C p}{6.25 \times 10^{-6} \times \pi \times \varepsilon_{o}}=\frac{t \times C p}{1.74 \times 10^{-16}}
$$

Whereas, the imaginary permittivity or dielectric loss, $\varepsilon_{i}$, is obtain from the value of dissipation factor, $D$.

Where, dissipation factor, $D$, 


$$
\begin{aligned}
D & =\frac{\varepsilon_{i}}{\varepsilon_{r}} \\
\varepsilon_{i} & =D \times \varepsilon_{r}
\end{aligned}
$$

With dissipation factor, $D$, is directly obtain from data of our measurement and $\varepsilon_{\mathrm{r}}$, is obtained from "Equation 3". Meanwhile, the dielectric dissipation factor, $\tan \delta$ or loss tangent of test material $D_{\mathrm{r}}$, can be obtained directly by measuring the dissipation factor from the "Equation 4".

\section{Results and discussion}

All figures are shown the behavior of Zinc oxide pellets that were sintered at different temperature and its behavior will determine dielectric constant of the pellets.

The relative permittivity $\varepsilon_{\mathrm{r}}$ of the pellets versus frequency of the applied LCR meter ranging from $1 \mathrm{kHz}$ until $5 \mathrm{MHz}$ with temperature as the function is shown in "Figure 2". Based on this, the relative permittivity $\varepsilon_{\mathrm{r}}$ of the pellets is decrease with increasing of frequency. The figure of sintered pellet at $800^{\circ} \mathrm{C}$ and $900^{\circ} \mathrm{C}$ are said to be in sequential order denotes, they possess the same behavior whereas as the temperature increase to $1000^{\circ} \mathrm{C}$, the behavior of the pellets seems to have changed. For pellet that was sintered at $700^{\circ} \mathrm{C}$, the figure shows that the sample has some defect.

For pellets sintered at $1000^{\circ} \mathrm{C}$, its $\varepsilon_{\mathrm{r}}$ is smaller than the pellets that are sintered at $900^{\circ} \mathrm{C}$ and $800^{\circ} \mathrm{C}$ due to increasing conductivity of the pellets. This is because, when those pellets were subjected to a sintering process at $1000^{\circ} \mathrm{C}$, volume of pore space between the particle is reduce. Thus, there are larger grain grows at the expense of smaller grains. Therefore, the energy barrier is reduced and rapidly increases the diffusion of atoms to another grain which means the diffusion coefficient is increase. Hence, the conductivity is increase with increasing of mobility, indicates the pellets sintered at $1000^{\circ} \mathrm{C}$ possess a conductor behavior.

For pellets that were sintered at $800^{\circ} \mathrm{C}$ and $900^{\circ} \mathrm{C}$, the relative permittivity of the pellets, $\varepsilon_{\mathrm{r}}$ are bigger than the other two pellets sintered at $700^{\circ} \mathrm{C}$ and $1000^{\circ} \mathrm{C}$ because at low temperature, therefore, the diffusion of atom is very slow due to an increasing of energy barrier. The diffusion coefficient is reduced which means the mobility and conductivity were decreased. Thus, the pellets can absorb and stored electrical potential, which indicate it possess dielectric behavior. Whereas for pellet subjected to $700^{\circ} \mathrm{C}$, it obtains $\varepsilon_{\mathrm{r}}$ lower than pellets sintered at $1000^{\circ} \mathrm{C}$. Based on theory, the pellets should obtain high $\varepsilon_{\mathrm{r}}$. This failure could be due to the existence of impurities during the preparation of the pellets. These impurities can diffuse and help carry the current and thus increasing the conductivity resulting small value relative permittivity of the pellets, $\varepsilon_{\mathrm{r}}$.

The imaginary part of permittivity or dielectric loss $\varepsilon_{\mathrm{i}}$ versus frequency of the applied field ranging from $1 \mathrm{kHz}$ until $5 \mathrm{MHz}$ with the temperature as the function is shown in "Figure 3". The pellet's dielectric loss, $\varepsilon_{\mathrm{i}}$ are decreasing with increasing frequency. Pellets that were sintered at $700^{\circ} \mathrm{C}, 800^{\circ} \mathrm{C}$ and $900^{\circ} \mathrm{C}$ have small dielectric loss comparable to $\varepsilon_{\mathrm{i}}$ of sintered pellet at $1000^{\circ} \mathrm{C}$. This indicates that they can store more energy due to small loss. Hence, they possess dielectric behavior. Meanwhile, $\varepsilon_{\mathrm{i}}$ for sintered pellets at $1000^{\circ} \mathrm{C}$ is high, indicates there are increasing of dielectric loss. Thus, it cannot store energy, therefore it possess conductor behavior.

The relative permittivity $\varepsilon_{\mathrm{r}}$ of the pellets versus temperature of the sintered pellets with the frequency of applied LCR meter as the function is shown in "Figure 4". This figure shows that relative permittivity of the pellets $\varepsilon_{\mathrm{r}}$, decreases with increased of frequency. This is because mechanisms of polarization have varying time response capability to an applied field frequency. For the frequency range between $1 \mathrm{kHz}$ and $1 \mathrm{MHz}$, the total contribution of polarization is arises from electronic displacement, ionic displacement, dipole orientation and space charge displacement. As the frequency increase at $1 \mathrm{MHz}$, the polarization of the molecule begins to lag the field reversals increasing the dielectric loss $\varepsilon_{\mathrm{i}}$, and decreasing relative permittivity $\varepsilon_{\mathrm{r}}$. Meanwhile, as the frequency decrease at $1 \mathrm{kHz}$, generates little loss, as the polarization mechanism is much slower than the field reversals.

The imaginary part of permittivity or dielectric loss $\varepsilon_{\mathrm{i}}$, versus temperature of the sintered pellets with the frequency of applied LCR meter as the function is shown in "Figure 5". With decreasing of frequency at $1 \mathrm{kHz}$ and $10 \mathrm{kHz}$, the molecules have large relaxation time with delayed polarization processes. Hence, the dielectric loss is small. Whereas, with increasing frequency at $1 \mathrm{MHz}$ and $100 \mathrm{kHz}$, the molecules are instantaneously polarized, thus the dielectric loss is high.

\section{Conclusion}

There are larger grain grows at the expense of smaller grains. The diffusion coefficient is increase due to the reduction of energy barrier and rapidly increases the diffusion of atoms to another grain. The diffusion coefficient is reduced when the mobility and conductivity were decreased. The pellets can absorb and stored electrical potential. The relative permittivity of the pellets $\varepsilon_{\mathrm{r}}$, decreases on term of increased of frequency due to the polarization, the molecules have large relaxation time when decreasing of frequency. It can be stored more energy because the dielectric loss is small. 


\section{References}

Askeland D. R. and Fulay P. P (2005) The Science and Engineering of Materials, Thomson-Engineering, Fifth Edition p.p. 156-694.

Griffiths D. J., Introduction to Electrodynamics $3^{\text {rd }}$ Ed (1999). Prentice Hall of India, p.p.160-192.

http://en.wikipedia.org/wiki/Zinc_oxide, available.

http://www.navbharat.co.in, (available 2008).

http://www.physorg.com, (available 2008).

http://www.spectrum.ieee.org, (Apr. 2008).

Marie Farnsworth and Charles H Kline, Zinc chemicals (1973) VG- Ex-lib (corporate) p.p. $36-57$.

Norton D. P., Heo Y. W., Ivill M. P., Ip K., Pearton S. J., Chisholm M. F., and Steiner T., (June 2004) ZnO growth, doping and processing, Material Today, p.p. 35- 36.

R.M. German, "Fundamentals of Sintering”, Engineering Materials Handbook, volume 4, (1991). Ceramics and Glasses, ASM International.

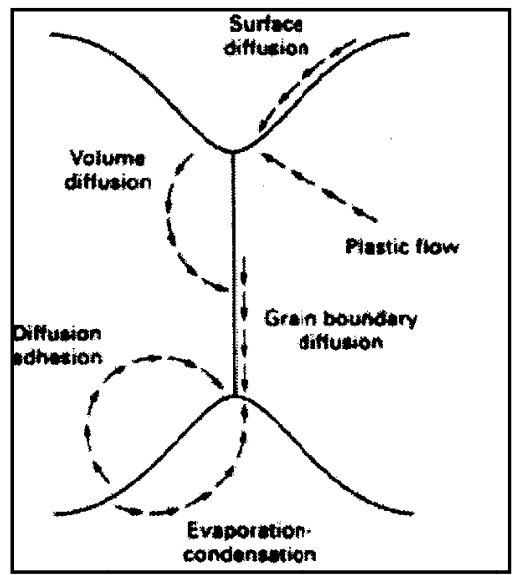

Figure 1. Mass flow paths in the neck region of two particles during sintering

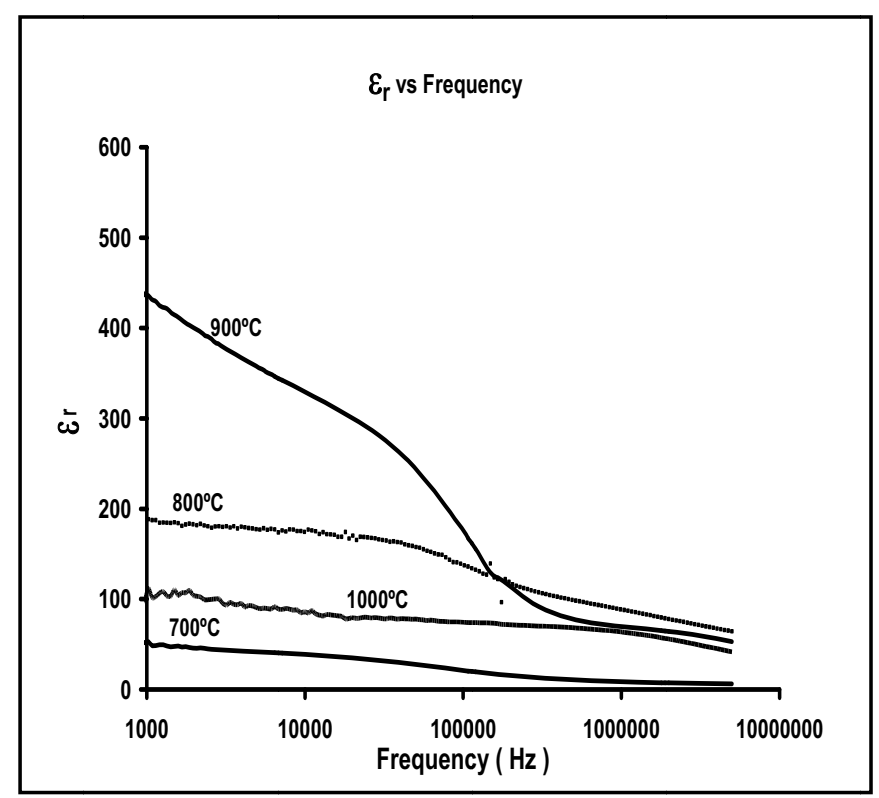

Figure 2. $\varepsilon_{\mathrm{r}}$ versus frequency $(\mathrm{Hz})$ 


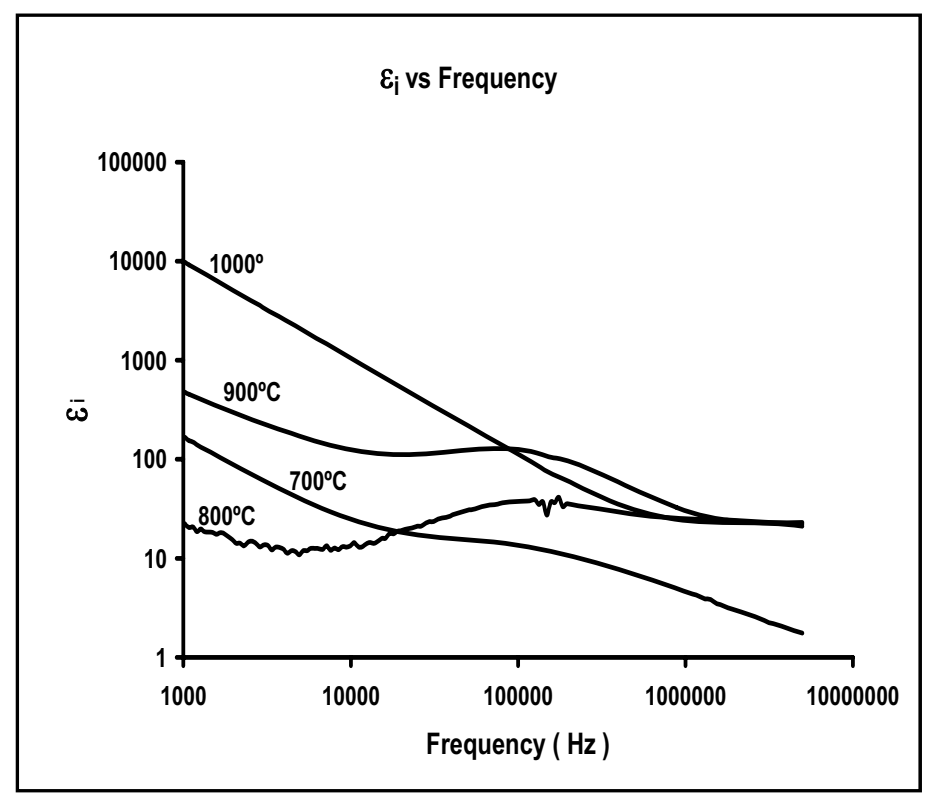

Figure $3 . \varepsilon_{\mathrm{i}}$ versus frequency $(\mathrm{Hz})$

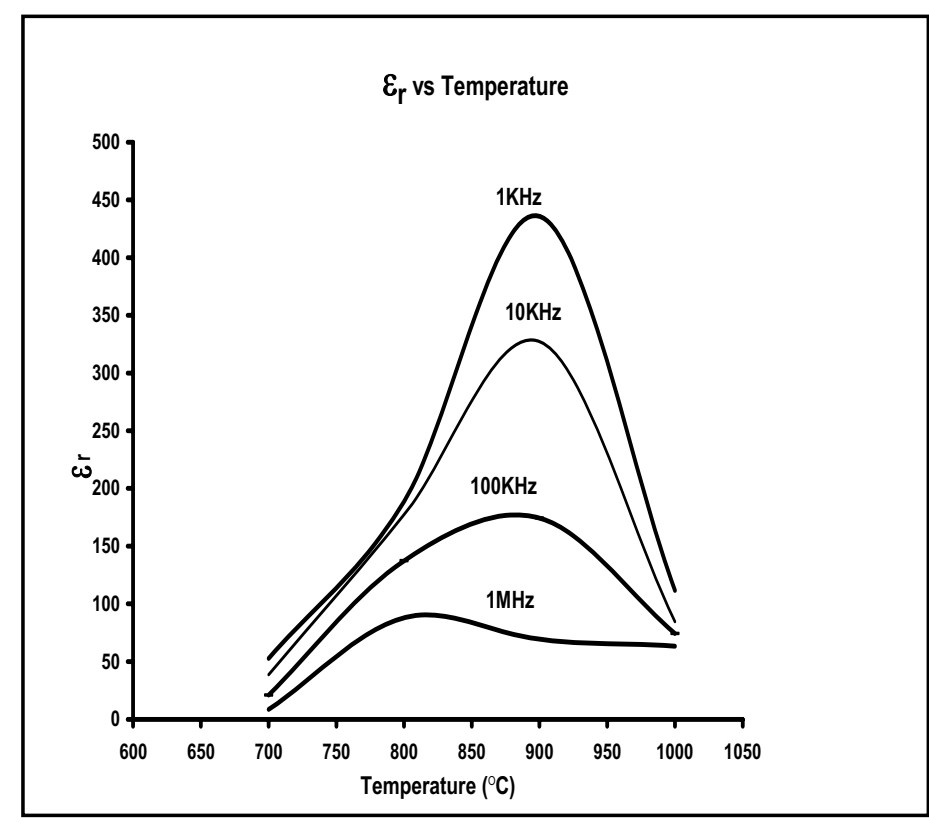

Figure 4. $\varepsilon_{\mathrm{r}}$ versus Temperature 


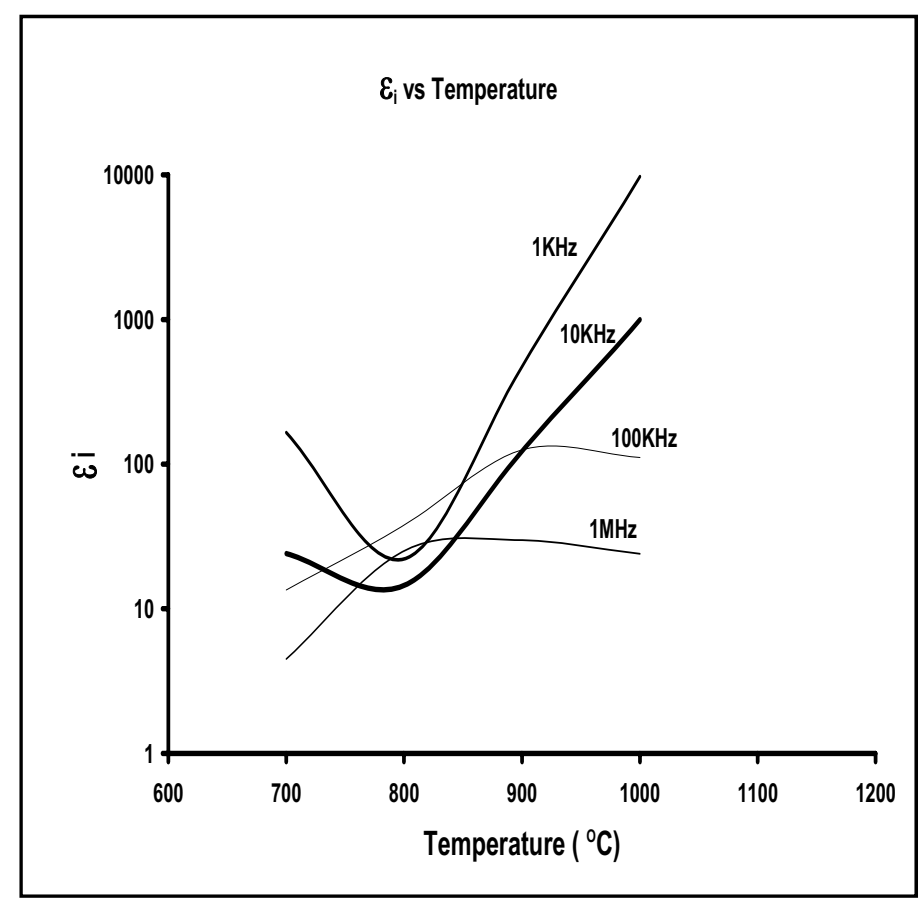

Figure 5. $\varepsilon_{\mathrm{i}}$ versus Temperature 